\title{
A NOTE ON $\delta$-CONTINUITY AND PROXIMATE FIXED POINTS FOR MULTI-VALUED FUNCTIONS
}

\author{
RAYMOND E. SMITHSON
}

1. Introduction. Let $X$ be a topological space and let $Y$ be a metric space with metric $d$. Let $f: X \rightarrow Y$ be a single valued function from $X$ into $Y$, and let $\delta>0$. Klee [2] has defined $f$ to be $\delta$-continuous if and only if for each $x \in X$ there is an open set $N(x)$ containing $x$ such that $d\left(f\left(x_{1}\right), f\left(x_{2}\right)\right)<\delta$ for all $x_{1}, x_{2} \in N(x)$.

Further, Klee [2] extended the notion of a fixed point to $\delta$-continuous functions and defined the proximate fixed point property. Then Yandl [9] obtained a number of results analogous to the results known for continuous functions. In particular he showed that the product of a countable number of chainable continua has the proximate fixed point property. The purpose of the present paper is to prove two proximate fixed point theorems for multifunctions.

2. Basic concepts. In the following let $F: X \rightarrow Y$ be a multi-valued function on a topological space $X$ into a metric space $Y$ and let $\delta>0$. If $A$ is a subset of $Y$, let $\mathrm{S}_{\delta}(A)=\{y \mid d(y, A)<\delta\}$ and let $\bar{S}_{\delta}(A)=\{y \mid d(y, A) \leqq \delta\}$, and denote the closure of a set $A$ by $A^{*}$.

Definitions. (1) The function $F$ is called lower $\delta$-continuous iff for each open set $V \subset Y$ the set $\left\{x \in X \mid F(x) \cap S_{\delta}(V) \neq \square\right\}$ is open.

(2) The function $F$ is called upper $\delta$-continuous iff for each closed set $A \subset Y$ the set $\left\{x \in X \mid F(x) \cap \bar{S}_{\delta}(A) \neq \square\right\}$ is closed.

(3) The function $F$ is called $\delta$-continuous iff it is both upper and lower $\delta$-continuous.

REMARK 1. The definitions given here do not correspond precisely to the definition given by Klee and used by Yandl, but there is a close relationship as will be shown below. These definitions were chosen because they are simple to state and easy to work with, whereas a more exact carryover of Klee's definition is cumbersome and presents some technical difficulties.

REMARK 2. If $F$ is a lower semicontinuous, upper semicontinuous, or continuous multi-valued function on $X$ into $Y$, then given any $\delta>0, F$ is lower $\delta$-continuous, upper $\delta$-continuous, or $\delta$-continuous respectively.

Proposition 1. If $F: X \rightarrow Y$ is $l$. $\delta-c$., if $\epsilon>0$, if $x \in X$ and if $y \in F(x)$, then there is an open set $N(x)$ containing $x$ such that

Received by the editors July 25, 1966. 
$d\left(y, F\left(x^{\prime}\right)\right)<\delta+\epsilon$ for all $x^{\prime} \in N(x)$. Consequently, if $F$ is $l . \delta-c$. for all $\delta>0$, then $F$ is lower semicontinuous (l.s.c.).

Proof. If $y \in F(x)$, then $S_{\epsilon}(y)$ is an open set and the set

$$
\left\{x^{\prime} \mid F\left(x^{\prime}\right) \cap S_{\delta}\left(S_{\epsilon}(y)\right) \neq \square\right\}
$$

is the required open set. Now let $V$ be open and let $y \in V \cap F(x)$. Pick $\epsilon$ such that $S_{2 \epsilon}(y) \subset V$, and let $\delta=\epsilon$. Then there is an open set $N(x)$ containing $x$ such that $d\left(y, F\left(x^{\prime}\right)\right)<2 \epsilon$. Hence $F\left(x^{\prime}\right) \cap V \neq \square$ for all $x^{\prime} \in N(x)$, and $F$ is 1.s.c.

Proposition 2. If $F(x)$ is compact for each $x \in X$, then $F u$. $\delta-c$. for each $\delta>0$ implies that $F$ is upper semicontinuous (u.s.c.).

Proof. Let $A$ be a closed subset of $Y$. Since $F(x)$ is compact and $A$ is closed, we have $\{x \mid F(x) \cap A \neq \square\}=\bigcap_{\delta>0}\left\{x \mid F(x) \cap \bar{S}_{\delta}(A) \neq \square\right\}$. Thus, $F$ is u.s.c.

Proposition 3. Let $F$ be $u . \delta-c$, let $\epsilon>0$ and let $x_{0} \in X$. Then there exists an open set $N\left(x_{0}\right)$ containing $x_{0}$ such that for each $x \in N\left(x_{0}\right)$ and each $y \in F(x)$, we have $d\left(y, F\left(x_{0}\right)\right)<\delta+\epsilon$.

Proof. Let $\eta=\delta+\epsilon$ and let $A=Y \backslash S_{\eta}\left(F\left(x_{0}\right)\right)$. Then

$$
\left\{x \mid F(x) \cap \bar{S}_{\delta}(A) \neq \square\right\}
$$

is closed, and hence, $N\left(x_{0}\right)=\left\{x \mid F(x) \cap \bar{S}_{\delta}(A)=\square\right\}$ is an open set containing $x_{0}$. Furthermore, if $x \in X$ and $y \in F(x)$ such that $d\left(y, F\left(x_{0}\right)\right)$ $\geqq \eta$, then $F(x) \cap A \neq \square$ and so $x \notin N\left(x_{0}\right)$. Consequently if $x \in N\left(x_{0}\right)$ and $y \in F(x)$, then $d\left(y, F\left(x_{0}\right)\right)<\delta+\epsilon$.

Remark 3. Propositions 1 and 3 show that the definitions used in this paper are related to the definition proposed by Klee. On the other hand, simple examples show that the two concepts are not equivalent.

3. The proximate fixed point property. The purpose of this section is to define the notion of the proximate fixed point property and to obtain generalizations of two fixed point theorems for multi-valued functions.

Definition. A metric space $X$ is said to have the proximate fixed point property (p.F.p.p.) for multi-valued functions iff for each $\epsilon>0$ there is a $\delta>0$ such that for each $\delta$-continuous multi-valued function $F: X>X$ there is a point $x \in X$ such that $d(x, F(x))<\epsilon$. 
Note. One defines the proximate fixed point property for $1 . \delta-\mathrm{c}$. and $u . \delta-c$. functions and for functions with restrictions of the image sets in an analogous way.

Proposition 4. If $X$ is compact and has the p.F.p.p. for functions $F$ such that $F(x)$ is compact for each $x$, then $X$ has the fixed point property (F.p.p.) for continuous multi-valued functions $F$ such that $F(x)$ is compact for each $x \in X$.

Proof. By Remark 2 each continuous function is $\delta$-continuous for each $\delta>0$. Thus, there exists a sequence $\left\{x_{n} \mid n \geq 1\right\}$ such that for any $\epsilon>0$ there is an $n$ such that $d\left(x_{n}, F\left(x_{n}\right)\right)<\epsilon$. Then, since $X$ and $F(x)$ are compact and $F$ is continuous, there is an $x_{0} \in X$ such that $x_{0} \in F\left(x_{0}\right)$.

The first theorem is a generalization of theorems of Hamilton [1], Ward [8], and Yandl [9].

Definition. A metric space $X$ is chainable iff given $\epsilon>0$ there exists a finite set $\mathcal{u}=\left\{U_{1}, \cdots, U_{n}\right\}$ of open sets such that: (i) $\cup \mathcal{U}=X$, (ii) $U_{i}^{*} \cap U_{j}^{*} \neq \square$ iff $|i-j| \leqq 1$, and (iii) $d\left(U_{i}\right)<\epsilon$ for all $i$.

The proof of Theorem 1 is patterned after the proof given by Ward [8].

THEOREм 1. Each chainable metric continuum has the p.F.p.p.

Proof. Let $X$ be a chainable metric continuum and let $\epsilon>0$ be given. Let $u$ be a finite collection of open sets satisfying properties (i), (ii), and (iii). Since $U_{i}^{*} \cap U_{j}^{*}=\square$ when $|i-j|>1, d\left(U_{i}^{*}, U_{j}^{*}\right)>0$ if $|i-j|>1$. Thus, let $\delta=\frac{1}{2} \min \left\{d\left(U_{i}^{*}, U_{j}^{*}\right)|| i-j \mid>1\right\}$, and let $F$ be any $\delta$-continuous function. Define the set $A$ by: $A=\{x \in X \mid$ for some $i, x \in U_{i}$ and for all $\left.\mathrm{j}<i, F(x) \cap S_{\delta}\left(U_{j}\right)=\square\right\}$. Note that $A$ is closed (since $F$ is $1 . \delta-c$.) and that $U_{1} \subset A$. Define the set $B$ by: $B=\left\{x \in X \mid\right.$ for some $i, x \in U_{i}$ and for some $\left.j<i, F(x) \cap \bar{S}_{\delta}\left(U_{j}^{*}\right) \neq \square\right\}$. Since $F$ is u. $\delta$-c., $B$ is closed. If $x \in X \backslash A$, then $F(x) \cap S_{\delta}\left(U_{j}\right) \neq \square$ for some $j<i$, and thus, $x \in B$. Therefore $X=A \cup B$. If $B=\square$, then for each $x \in U_{n}, F(x) \subset U_{n}$, and hence, $d(x, F(x))<\epsilon$. So suppose that $B \neq \square$. Thus, since $X$ is connected and $A, B$ are closed, there is an $x_{0} \in A \cap B$. Let $i$ be the least index such that $x_{0} \in U_{i}$. Then $F\left(x_{0}\right)$ $\cap S_{\delta}\left(U_{j}\right)=\square$ for all $j<i$. Note if $F\left(x_{0}\right) \cap \bar{S}_{\delta}\left(U_{i-1}^{*}\right) \neq \square$, then $F\left(x_{0}\right)$ $\cap U_{i} \neq \square$ and we have $\left.d\left(x_{0}, F\left(x_{0}\right)\right)\right)<\epsilon$. Furthermore, if $F\left(x_{0}\right)$ $\cap\left(U_{i} \cup U_{i+1}\right)=\square$, then $F\left(x_{0}\right) \subset \cup\left\{U_{j}^{*} \backslash U_{i} \mid j>i+1\right\}=C$. Hence, if $j^{\prime} \leqq i, d\left(F\left(x_{0}\right), U_{j^{\prime}}^{*}\right) \geqq d\left(C, U_{j^{\prime}}^{*}\right) \geqq 2 \delta$. But $x_{0} \in B$ implies that

$$
d\left(F\left(x_{0}\right), U_{j^{\prime}}^{*}\right) \leqq \delta
$$

for some $j^{\prime} \leqq i$. Thus, $F\left(x_{0}\right) \cap\left(U_{i} \cup U_{i+1}\right) \neq \square$. Finally, the above also implies that $x_{0} \in U_{i} \cap U_{i+1}$, if $F\left(x_{0}\right) \cap U_{i}=\square$, and so $d\left(x_{0}, F\left(x_{0}\right)\right)<\epsilon$. 
Note. In general a countable (or finite) product of chainable continua will not have the p.F.p.p. Indeed, Strother [5] has exhibited a continuous multi-valued function on the unit disc into itself which does not have a fixed point.

The second theorem is a generalization of a theorem of Plunkett's [4] on dendrites. Recall that a dendrite is a metric continuum such that any two points can be separated by the omission of a third point. We shall use the partial order structure of a dendrite that was developed by Ward [6]. Let $X$ be a dendrite and let $e$ by any point of $X$. Define the partial order $P$ by: $P=\{(x, y) \in X \times X \mid x=e, x$ separates $e$ and $y$, or $x=y\}$. Then $P$ is a partial order with minimal element $e, P=P^{*}$ in the product topology on $X \times X$, and the sets $x P=\{y \mid(x, y) \in P\}$ and $P x=\{y \mid(y, x) \in P\}$ are closed. Further, the sets $x P \backslash x$ are open and the sets $P x \cap P y$ are compact, nonempty, connected chains. We shall write $x \leqq y$ iff $(x, y) \in P$. Also, results of Nachbin [3] and Ward [6], [7] show that each chain in $X$ has both a supremum and an infimum and that each closed set has minimal and maximal elements.

ThEOREM 2. Each dendrite has the p.F.p.p.

Proof. Let $X$ be a dendrite and let $\epsilon>0$. Let $e \in X$, and let $P$ be the above partial order with minimal element $e$. Since $X$ is uniformly locally connected, for each $\eta>0$ there is a $\delta_{\eta}>0$ such that if $d(x, y)$ $<\delta_{\eta}$, then $x$ and $y$ are contained in a connected set with diameter less than $\eta$. Let $\eta=\epsilon$, let $\delta=\delta_{\eta / 2}$ and let $F$ be a $\delta$-continuous function on $X$ in to $X$. If $e \in F(e)$, we are done. Thus, the set $\{x \mid F(x) \cap x P \neq \square\}$ is nonempty. Let $C$ be a maximal chain in $\{x \mid F(x) \cap x P \neq \square\}$, and let $x_{0}=\sup C$. We claim that $x_{0} \in C$. Let $U$ be a convex open set containing $x_{0}$ such that $d(U)<\delta$, and set $A=U\{F(x) \cap x P \mid x \in C \cap U\}$. Then, since $F$ is u. $\delta-$ c., $x_{0} \in\left\{x \mid F(x) \cap \bar{S}_{\delta}\left(A^{*}\right) \neq \square\right\}$; thus, let $x_{1} \in F\left(x_{0}\right)$ and $x_{2} \in A^{*}$ such that $d\left(x_{1}, x_{2}\right) \leqq \delta$. First note that if $x_{2} \in U^{*}$, there is an $x^{\prime} \in U$ and $x^{\prime \prime} \in F\left(x^{\prime}\right)$ such that $d\left(x^{\prime}, x^{\prime \prime}\right)<2 \delta$, and thus, $d\left(x^{\prime}, F\left(x^{\prime}\right)\right)<\epsilon$. On the other hand, if $x_{2} \notin U^{*}$ and if $x_{0} \nless x_{2}$, there is an open set $V$ such that $x_{2} \in V$ and if $x^{\prime} \in V, x^{\prime \prime} \in U$, then $x^{\prime \prime} \varangle x^{\prime}$, but this contradicts the definitions of $A$ and $C$. Then if $x_{0} \nless x_{1}, x_{0}$ separate $x_{1}$ and $x_{2}$, but by the uniformly local connectedness property $x_{1}$ and $x_{2}$ are contained in a connected set of diameter less than $\epsilon$ which implies that $d\left(x_{0}, F\left(x_{0}\right)\right)<\epsilon$. Consequently we conclude that $x_{0} \in C$. Now let $x_{1}$ be a minimal element of the closure of $x_{0} P \cap F\left(x_{0}\right)$ and let $V=x_{1} P \backslash x_{1}$. Since $F$ is $1 . \delta-c_{\text {. }}$, the set

$$
\left\{x \mid F(x) \cap S_{\delta}(V) \neq \square\right\}
$$


is open; thus, there is an element $x_{2}$ such that $x_{0}<x_{2}<x_{1}$ and such that $F\left(x_{2}\right) \cap S_{\delta}(V) \neq \square$. Let $x_{3} \in F\left(x_{2}\right) \cap S_{\delta}(V)$. Then, since $C$ was a maximal chain, $x_{2} \nless x_{3}$, and thus, $x_{2}$ separates $x_{3}$ and some point $x_{1}^{\prime}$ of $V$ with $d\left(x_{1}, x_{3}\right)<\delta$. This implies that $x_{3}$ and $x^{\prime}$ are contained in a connected set with diameter less than $\epsilon$, but $x_{2}$ must also be in this set, which implies that $d\left(x_{2}, F\left(x_{2}\right)\right)<\epsilon$. So, in any case, we have a point $x \in X$ such that $d(x, F(x))<\epsilon$, and the theorem is proved.

Finally, combining Proposition 4, Theorem 2, and a result of Plunkett's theorem [4], we get

Corollary. A Peano space has the p.F.p.p. iff it is a dendrite.

\section{REFERENCES}

1. O. H. Hamilton, A fixed point theorem for pseudo-arcs and certain other metric continua, Proc. Amer. Math. Soc. 2 (1951), 173-174.

2. V. Klee, Stability of the fixed point property, Colloq. Math. 8 (1961), 43-46.

3. L. Nachbin, Topology and order, Van Nostrand, Princeton, N. J., 1965.

4. R. L. Plunkett, A fixed point theorem for continuous multi-valued transformations, Proc. Amer. Math. Soc. 7 (1956), 160-163.

5. W. L. Strother, Fixed points, fixed sets and M-retracts, Duke Math. J. 22 (1955), 551-556.

6. L. E. Ward, Partially ordered topological spaces, Proc. Amer. Math. Soc. 5 (1954), 144-161.

7. —_, A note on dendrites and trees, Proc. Amer. Math. Soc. 5 (1954), 992-993.

8. — A fixed point theorem, Amer. Math. Monthly 65 (1958), 271-272.

9. A. L. Yandl, On the proximate fixed point property, Notices Amer. Math. Soc. 12 (1965), 589.

UNIVERSITY OF WYOMING 